\title{
Acute Tumor Lysis Syndrome - a Rare Complication in the Treatment of Solid Tumors
}

\author{
Bertrand Coiffier
}

Department of Hematology, Hospices Civils de Lyon and Université Lyon-1, Lyon, France

Tumor lysis syndrome (TLS) with acute renal failure is a very rare complication of the treatment of solid tumors. Most incidents of TLS occurred in hematological malignancies, acute leukemia or lymphoma. In these diseases, it may occur spontaneously in a tumor with rapid proliferation or after beginning treatment $[1,2]$. Recently, cases of TLS have been described in more indolent proliferations such as chronic lymphocytic leukemia or multiple myeloma because of the increased efficacy of new molecules in these diseases [3, 4]. Report of TLS in solid tumors is rare but can occur and TLS is continuously reported as a unexpected complication of treatments because oncologists rarely think about it before treating patients [5-8].

TLS is a serious condition leading to the death of the patient in $20-50 \%$ of cases if undiagnosed or if diagnosed too late. The best treatment is prevention, that is to look for the first biologic abnormalities characterizing TLS (hyperuricemia, hyperphosphatemia, increased creatinine level) and to treat it before its full expression [9]. Systematic prevention is used in some leukemias or lymphomas, however, TLS occurrence is too low to do such a systematic prevention in solid tumors, except perhaps in some situations like the cases reported by Wang and Chen [10] in this issue of ONKOLOGIE.

TLS is caused by the lysis of malignant cells by cytotoxic chemotherapy or other anti-cancer procedures, leading to a rapid release of the intracellular contents into the blood stream including potassium, phosphate, and purines from the breakdown of nuclear proteins. Secondary hypocalcemia, hyperkalemia and hyperphosphatemia lead to gastrointestinal disturbances, neuromuscular effects and cardiovascular consequences. Purines are catabolized to hypoxanthine, then xanthine, and finally in the liver to uric acid by the enzyme xanthine oxidase. The precipitation of uric acid in excess contributes to impaired renal function; acute renal failure represents the most frequent and serious clinical consequence of TLS-related hyperuricemia [11]. Risk factors include certain types of malignancy, some tumor-related factors such as high numbers of circulating tumor cells and a large tumor burden, the presence of individual factors, such as pre-existing renal insufficiency, and the type and intensity of anti-cancer regimen used [11].

A recent survey of TLS in solid tumors in one institution found an incidence of less than $0.3 \%$ [6]. TLS has been observed in small cell lung cancer, breast cancer, germ cell tumors, melanoma, sarcomas, hepatocellular carcinoma, ovarian cancer, colorectal cancer, and prostate cancer with various types of chemotherapy, radiation therapy or anti-cancer procedures [8]. Why TLS is rarely observed in solid tumors, even in tumors with the same growth kinetics and sensitivity to chemotherapy as hematological cancer, is not really understood. In solid tumors, contrarily to what is observed in hematological cancers, TLS is often observed a few days after the initiation of treatment, when the patient has left the hospital [5]. This may explain why the mortality associated with TLS in solid tumors is higher than in hematological cancers [12,13]. Risk factors for TLS in patients with solid tumors are high tumor volume with metastatic presentation, liver metastasis, and the classical risk factors (high LDH level, high uric acid level, pre-existent renal insufficiency) [8].

The standard management strategy for treating TLS is based on hydration and decreasing uric acid concentration. This may be obtained with rasburicase that lyses uric acid into allantoin, a much more soluble product [14, 15]. Several studies have demonstrated that rasburicase is generally welltolerated, although severe adverse effects were reported such as hypersensitivity reactions [14]. Allopurinol has a great efficacy for preventing TLS in high-risk patients but has a limited activity when TLS has already developed and the creatinine level has increased [1,2,9].

In conclusion, TLS is a rare event in patients with solid tumors but it may occur more frequently in the future because of the very active armamentarium that is currently developed for these patients. It usually occurs a few days after treatment but is unpredictable. Because of that the mortality rate associated with TLS in solid tumors is high. It is easily prevented with allopurinol and must be treated with hydration plus

\section{KARGER}

Fax +497614520714

Information@Karger.de

www.karger.com (c) 2010 S. Karger GmbH, Freiburg

Accessible online at:

www.karger.com/onk
Prof. Bertrand Coiffier

Hématologie, Centre Hospitalier Lyon-Sud

69310 Pierre-Benite, France

Fax +33478 86-4355

bertrand.coiffier@chu-lyon.fr 
rasburicase. Although the reported number of TLS cases occurring in solid tumors is low, it may be that it is underdiagnosed or unsuspected in most solid tumor patients. Physi- cians must be alerted on the possibility of TLS, even in solid tumors, so they can preemptively manage it.

\section{References}

1 Coiffier B, Altman A, Pui CH, Younes A, Cairo MS: Guidelines for the management of pediatric and adult tumor lysis syndrome: an evidence-based review. J Clin Oncol 2008:26:2767-2778.

2 Cairo MS, Coiffier B, Reiter A, Younes A: Recommendations for the evaluation of risk and prophy laxis of tumour lysis syndrome (TLS) in adults and children with malignant diseases: an expert TLS panel consensus. Br J Haematol 2010;149:578-586.

3 Coiffier B, Riouffol C: Management of tumor lysis syndrome in adults. Expert Rev Anticancer Ther 2007;7:233-239.

4 Sezer O, Vesole DH, Singhal S, et al.: Bortezomibinduced tumor lysis syndrome in multiple myeloma. Clin Lymphoma Myeloma 2006;7:233-235.

5 Baeksgaard L, Sorensen JB: Acute tumor lysis syndrome in solid tumors - a case report and review of the literature. Cancer Chemother Pharmacol 2003;51:187-192.
6 Mott FE, Esana A, Chakmakjian C, Herrington JD: Tumor lysis syndrome in solid tumors. Support Cancer Ther 2005;2:188-191.

7 Pinder EM, Atwal GS, Ayantunde AA, et al. Tumour lysis syndrome occurring in a patient with metastatic gastrointestinal stromal tumour treated with glivec (imatinib mesylate, gleevec, STI571). Sarcoma 2007;2007:82012.

8 Gemici C: Tumour lysis syndrome in solid tumours. Clin Oncol (R Coll Radiol) 2006;18:773-780.

9 Mughal TI, Ejaz AA, Foringer JR, Coiffier B: An integrated clinical approach for the identification, prevention, and treatment of tumor lysis syndrome. Cancer Treat Rev 2010;36:164-176.

10 Wan K, Chen Z: Acute tumor lysis syndrome after transarterial chemoembolization for welldifferentiated hepatocellular carcinoma with neuroendocrine features. Onkologie 2010;33: DOI: $10.1159 / 000319695$
11 Michallet AS, Tartas S, Coiffier B: Optimizing management of tumor lysis syndrome in adults with hematologic malignancies. Support Cancer Ther 2005;2:159-166.

12 Drakos P, Bar-Ziv J, Catane R: Tumor lysis syndrome in nonhematologic malignancies. Report of a case and review of the literature. Am J Clin Oncol 1994;17:502-505.

13 Castro MP, VanAuken J, Spencer-Cisek P, Legha S, Sponzo RW: Acute tumor lysis syndrome associated with concurrent biochemotherapy of metastatic melanoma: a case report and review of the literature. Cancer 1999;85:1055-1059.

14 Coiffier B, Mounier N, Bologna S, et al.: Efficacy and safety of rasburicase (recombinant urate oxidase) for the prevention and treatment of hyperuricemia during induction chemotherapy of aggressive non-Hodgkin's lymphoma: results of the GRAAL1 study. J Clin Oncol 2003;21:4402-4406.

15 Hochberg J, Cairo MS: Tumor lysis syndrome: current perspective. Haematologia 2008;93:9-13. 